\title{
LETTERS
}

\section{Sitaxentan-induced hepatic failure in two patients with pulmonary arterial hypertension}

\section{To the Editor:}

Sitaxentan sodium is an endothelin receptor antagonist (ERA) recently approved for the management of pulmonary arterial hypertension (PAH) [1]. While hepatotoxicity is a recognised side-effect of treatment with the ERA bosentan, it is considered to be less common with sitaxentan [2-4]. We recently admitted two patients with severe hepatotoxicity on sitaxentan to the Pulmonary Hypertension Unit at the Mater Misericordiae University Hospital (Dublin, Ireland).

The first patient was a 47-yr-old male with PAH associated with congenital heart disease. He had a past history of ventricular septal defect closure at 12 yrs of age and was referred to our service with a history of gradual diminishing exercise capacity. His right heart catherisation demonstrated a pulmonary artery systolic pressure of $80 \mathrm{mmHg}$ and a normal pulmonary capillary wedge pressure. He was commenced on sitaxentan $100 \mathrm{mg}$ q.d. Initial liver function tests (LFTs) were normal, and the patient continued to undergo monthly LFT monitoring. At 3 months, he was experiencing an improvement in his symptoms and 6-min walking distance. However, 1 month later, he became generally unwell, with nausea, vomitting, anorexia and weight loss. He denied abdominal pain or pruritis. Physical examination was notable for icterus without evidence of hepatomegaly. The course of the patient's LFTs are shown in figure 1a. Treatment with sitaxentan was immediately discontinued. The patient was on no other hepatotoxic medications. At review 2 weeks later, his clinical condition was unchanged and LFTs had deteriorated; he was admitted to the hospital. Initial haematological tests showed a haemoglobin level of $15.7 \mathrm{~g} \cdot \mathrm{dL}^{-1}$, a white cell count of $6.54 \times 10^{9} \cdot \mathrm{L}^{-1}$, a platelet count of $158 \times 10^{9} \cdot \mathrm{L}^{-1}$, erythrocyte sedimentation rate $2 \mathrm{~mm}$ in the first hour, a C-reactive protein level of $5.8 \mathrm{~g} \cdot \mathrm{L}^{-1}$, and normal renal function tests. Auto-antibody screening was negative apart from parietal cell antibody positivity. $\alpha_{1}$-Antitrypsin and copper levels were within normal limits. Virology serology, including hepatitis A, B and C, HIV and anti-cytomegalovirus were all negative. Epstein-Barr virusassociated nuclear antigen immunoglobulin $G$ was positive, indicating previous exposure. The patient was commenced on vitamin K for his coagulopathy; however, his INR still peaked at 1.6. Liver ultrasound showed a normal liver in both size and echogenecity, with no evidence of intrahepatic or common bile duct dilation. The portal vein was patent. As part of diagnostic investigations, the patient underwent transjugular liver biopsy. This was consistent with severe acute drug-induced liver injury (fig. 2). Ursodeoxycholic acid was commenced. The patient's

For editorial comments see page 529. clinical condition and liver function testing gradually improved over the following months (fig. 1a).

The second patient was a 70-yr-old female with severe idiopathic PAH. She was commenced on sitaxentan $100 \mathrm{mg}$ a day at the end of July 2008, with monthly LFT monitoring. At the time that sitaxentan was commenced, she was also taking aspirin $75 \mathrm{mg}$ a day and the antihypertensives doxazosin XL $8 \mathrm{mg}$ and valsartan/hydrochlorothiazide $80 / 12.5 \mathrm{mg}$ once a day. Frusemide $20 \mathrm{mg}$ a day commenced shortly after the ERA was initiated. However, after 3 months, the patient's LFTs increased and continued despite discontinuing the sitaxsentan in midNovember (fig. 1b). The patient's aspartate aminotransferase
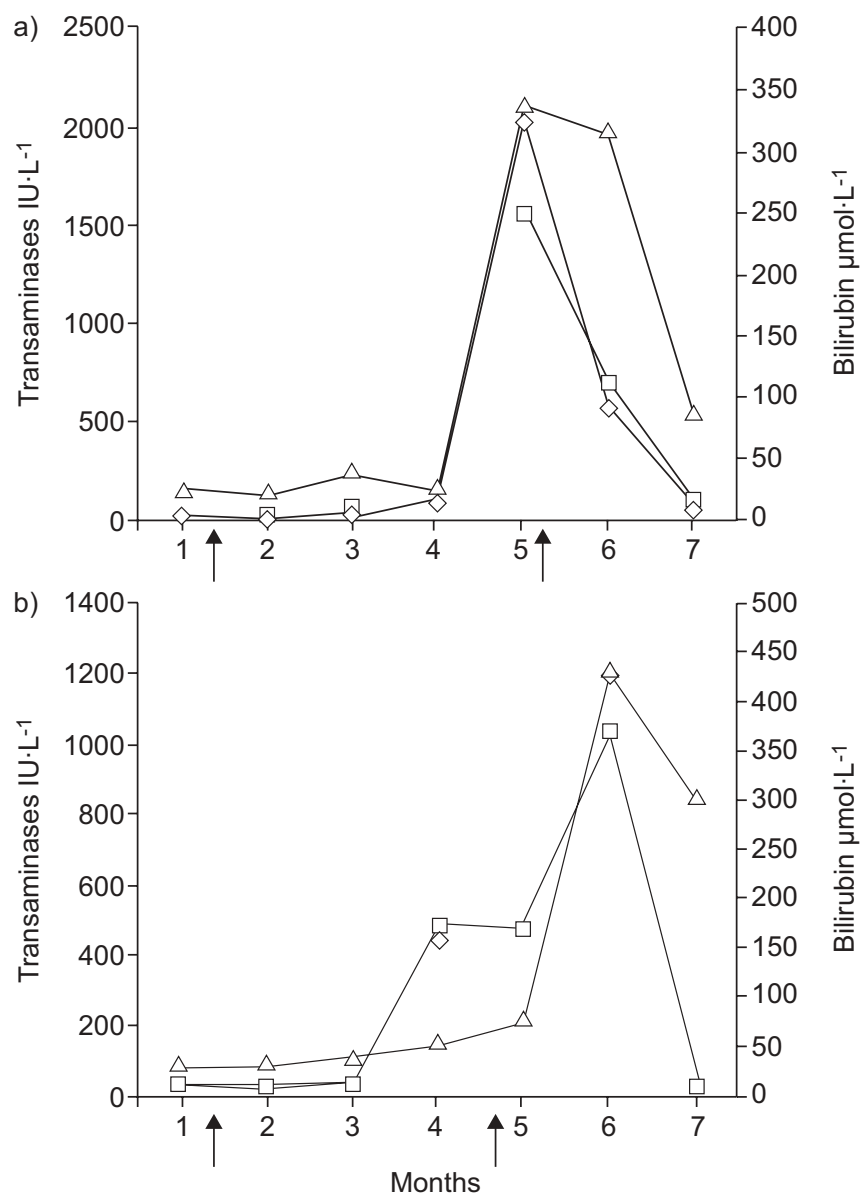

FIGURE 1. Change in liver function tests over time from commencement of sitaxentan therapy in months in the a) first and b) second patients. The courses of bilirubin $(\triangle)$, aspartate aminotransferase $(\diamond)$ and alanine aminotransferase $(\square)$ are shown. The arrows mark the beginning and end of sitaxentan therapy. 


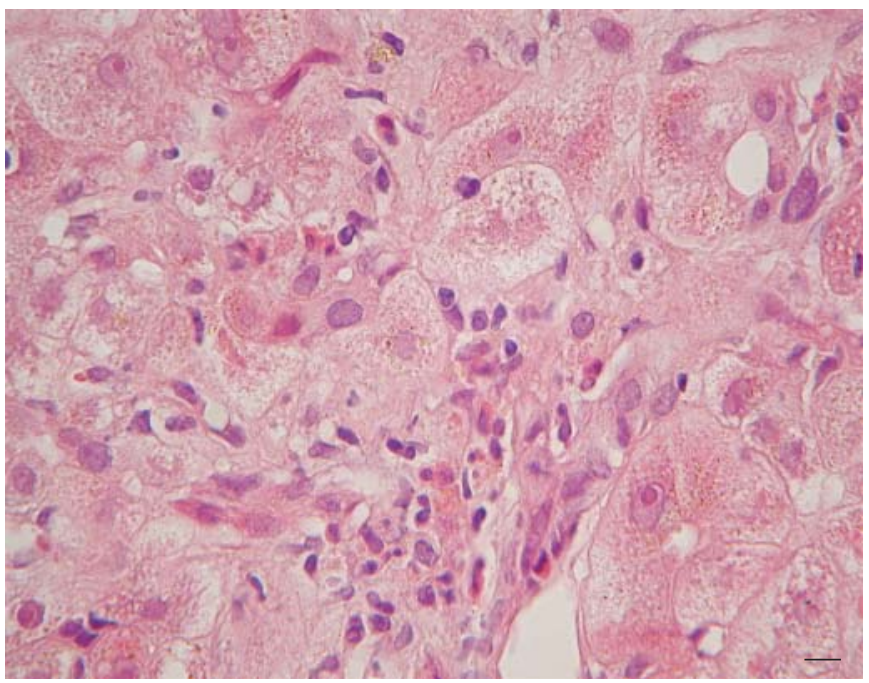

FIGURE 2. Liver biopsy of patient 1 demonstrating typical features of a drug reaction i.e. ballooning degeneration of hepatocytes, bile stasis and infiltration by eosinophils. Haematoxylin and eosin staining. Scale bar $=2 \mu \mathrm{m}$.

(AST) and alanine aminotransferase (ALT) peaked at 1,198 IU and 1,040 IU respectively, while her bilirubin peaked at $426 \mu \mathrm{mol} \cdot \mathrm{L}^{-1}$, with her INR reaching 1.6 off anticoagulation. Two months after the drug was discontinued, the AST and ALT had returned to normal but the bilirubin remained $>200 \mathrm{umol} \cdot \mathrm{L}^{-1}$. Both hepatitis B surface antigen and hepatitis $\mathrm{C}$ virus were negative; however, as in the first case, the patient's parietal cell antibody was positive. Her liver ultrasound revealed a normal liver size and echogenecity. A liver biopsy (fig. 3) showed a moderate portal and parenchymal lymphocytic infiltrate with scattered eosinophils and extensive active hepatocyte injury. Areas of confluent necrosis were identified. Although the patient recovered from the acute liver injury, she was readmitted in January 2009 with lobar pneumonia and persistant jaundice, and died of respiratory failure.

While initial studies with sitaxentan $300 \mathrm{mg}$ demonstrated its potential to cause liver injury following the death of one patient [3], a follow-up study using sitaxentan at a lower dose of $100 \mathrm{mg}$ q.d. caused no significant elevation of liver enzymes [5]. However, an accumulation of sitaxentan may occur as a result of nonlinear pharmacokinetics previously demonstrated during drug development [3]. Accumulation may explain the delay in time to injury and the prolonged recovery following discontinuation of the drug.

The liver injury suffered by the two patients presented in this study was severe. The transaminases peaking at $>30$ times the upper limit of normal. In addition, the pattern of hepatic injury was mixed with significant elevation of both bilirubin and alkaline phosphatase also observed. Both cases occurred within the first 12 weeks of treatment and resulted in a protracted period of jaundice.

In conclusion, we have presented two cases of fulminant hepatic failure from sitaxentan therapy for PAH. These cases

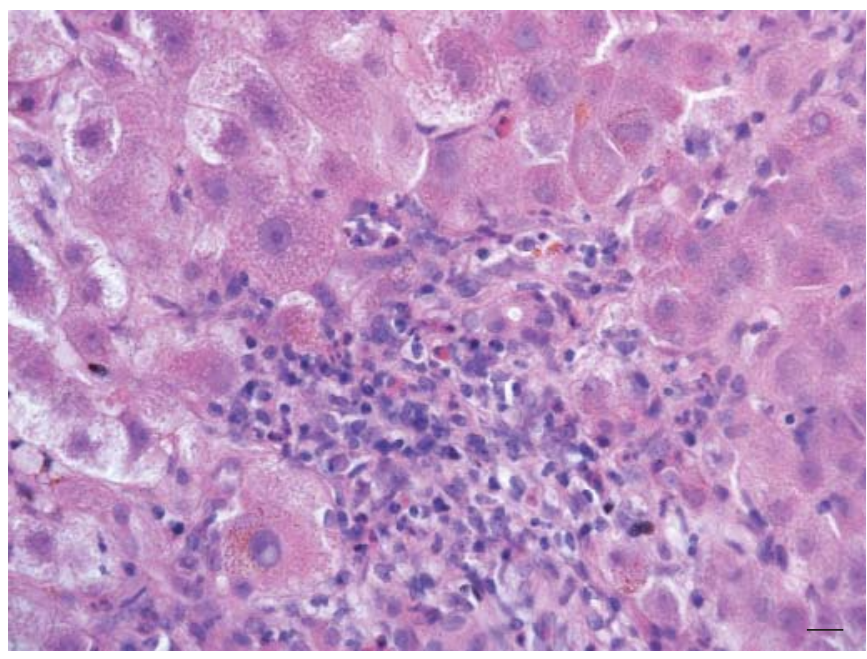

FIGURE 3. Liver biopsy of patient 2 showing moderate portal and parenchymal lymphocytic infiltrate with scaterred eosinophils and extensive active hepatocyte injury. Haematoxylin and eosin staining. Scale bar $=2 \mu \mathrm{m}$.

emphasise the importance of monthly liver function testing in patients treated with these agents.

\section{A. Lavelle, R. Sugrue, G. Lawler, N. Mulligan, B. Kelleher, D.M. Murphy and S.P. Gaine}

The Mater Misericordiae University Hospital, Dublin, Ireland.

Correspondence: S.P. Gaine, The Pulmonary Hypertension Unit, The Mater Misericordiae University Hospital, Eccles Street, Dublin 7, Ireland. E-mail: sgaine@o2.ie

Statement of Interest: A statement of interest for S.P. Gaine can be found at www.erj.ersjournals.com/misc/statements.dtl

\section{REFERENCES}

1 O'Callaghan DS, Gaine SP. Sitaxsentan: an endothelin-A receptor antagonist for the treatment of pulmonary arterial hypertension. Int J Clin Pract 2006; 60: 475-481.

2 Benza RL, Mehta S, Keogh A, et al. Sitaxsentan treatment for patients with pulmonary arterial hypertension discontinuing bosentan. J Heart Lung Transplant 2007; 26: 63-69.

3 Barst RJ, Rich S, Widlitz A, et al. Clinical efficacy of sitaxsentan, an endothelin-A receptor antagonist, in patients with pulmonary arterial hypertension: open-label pilot study. Chest 2002; 121: 18601868.

4 Humbert M, Segal ES, Kiely DG, et al. Results of European postmarketing surveillance of bosentan in pulmonary hypertension. Eur Respir J 2007; 30: 338-344.

5 Barst RJ, Langleben D, Frost A, et al. Sitaxsentan therapy for pulmonary arterial hypertension. Am J Respir Crit Care Med 2004 169: 441-447. 